\title{
BLOQUEIO ANESTÉSICO DO NERVO OCCIPITAL MAIOR NA PROFILAXIA DA MIGRÂNEA
}

\author{
Elcio Juliato Piovesan', Lineu Cesar Werneck², Pedro André Kowacs', \\ Claudio Esteves TatsuP', Marcos Christiano Lange ${ }^{3}$, Maurice Vincent"
}

\begin{abstract}
RESUMO - Na fisiopatologia da enxaqueca muitas estruturas estão envolvidas, sendo que o nervo trigêmeo pode ser considerado a estrutura principal. Com o objetivo de determinar a influência do nervo occipital maior (NOM) sobre o comportamento da enxaqueca, estudamos 37 pacientes que apresentavam crises de enxaqueca. Utilizando-se de um estudo duplo cego "cruzado" os pacientes foram submetidos a infiltração do NOM com bupivacaína 0,5\% (BP) e soro fisiológicos 0,9\% (SF), os efeitos clínicos após os bloqueios anestésicos foram avaliados: subjetivamente através da escala visual analítica para dor e objetivamente determinou-se os limiares de percepção dolorosa. A comparação entre os dois grupos (BP-SF) e (SF-BP) mostrou que: o número e a duração das crises em todos os momentos do estudo não mudaram; a intensidade das crises no grupo (BP$\mathrm{SF})$ foi menor somente depois da segunda infiltração $(P=0,020)$, em todos os outros momentos não se observaram alterações significativas. Concluímos que o bloqueio anestésico com BP sobre o NOM não altera o número e a duração das crises de migrânea, porém promove uma redução média na intensidade das crises 60 dias após a sua infiltração. Os resultados mostrados sugerem que o NOM participa ativamente sobre a modulação nociceptiva durante as crises de enxaqueca sem aura.
\end{abstract}

PALAVRAS-CHAVE: bloqueio anestésico, nervo occipital maior, enxaqueca, profilaxia.

Anesthetic blockade of the greater occipital nerve in migraine prophylaxis

\begin{abstract}
Migraine comprises a great many encephalic structures in its pathophysiology with the trigeminal nerve (TN) type being one of the main ones. For the purpose of determining a possible influence of the greater occipital nerve (GON) on migraine behavior, 37 patients who showed this pathology were studied. Using a double blind "cross over" group and submitting those patients to a GON infiltration with bupivacaina $0.5 \%$ (BP) and physiological serum $0.9 \%$ (PS), the clinical effects were evaluated: subjectively, through a pain analytical visual scale; objectively, by determining the threshold of pain perception (algometry). The comparison between the two groups (BP-PS) and (PS-BP) has shown that the number and duration of the attacks did not show significant statistical differences during the study. The intensity of the attacks was lower in group (BP-PS) only after the second infiltration $(p=0.020)$, in the other moments no differences have been observed between the groups. The conclusion is that the anesthetic blockage with BP on the GON does not change the number of crises and their duration, but it does provokes an intensity reduction after 60 days from the infiltration. The results shown here suggest that GON participates in the cranial nociceptive modulation during crises of migraine without aura.
\end{abstract}

KEY WORDS: blockade, greather occipital nerve, migraine, prophylaxis.

O nervo occipital maior (NOM) é um dos responsáveis pela inervação sensorial cutânea da região occipital e suboccipital'. Pesquisas recentes mostraram que ele também participa ativamente na modulação nociceptiva encefálica, atuando diretamente, em conjunto com o nervo trigêmeo (NT), sobre neurônios nociceptivos secundários, localizados ao nível do tronco cerebral ${ }^{2}$. A influência do NOM sobre os mecanismos fisiológicos da migrânea não foram até o momento, adequadamente esclarecidos.

Neste estudo procurou-se determinar o comportamento clínico da migrânea após o bloqueio anestésico do NOM como conduta profilática.

\section{MÉTODO}

Foram admitidos neste estudo sessenta e três pacien-

Unidade de Cefaléias, Especialidade e Serviço de Neurologia do Departamento de Clínica Médica, Hospital de Clínicas, Universidade Federal do Paraná (UFPR), Curitiba PR, Brasil: ${ }^{1}$ Neurologista; ${ }^{2}$ Professor Titular; ${ }^{3}$ Medico; ${ }^{4}$ Professor Adjunto (Setor de Cefaléias, Serviço de Neurologia, Hospital Universitário Clementino Fraga Filho, Universidade Federal do Rio de Janeiro).

Recebido 20 Dezembro 2000, recebido na forma final 13 Março 2001. Aceito 17 Março 2001. 
tes da unidade de cefaléias, que apresentavam crises que preenchiam os critérios para a migrânea sem aura propostos pela International Headache Society $(\mathrm{IHS})^{3}$ e que possuiam idade superior a 18 e inferior a 55 anos, presença de uma ou mais crises ao mês, ausência de história pregressa de traumatismo cranio-encefálico ou raquimedular, ausência de patologias osteoarticular, ligamentar ou muscular na coluna cervical e ausência de qualquer outro tipo de cefaléia nos últimos 12 meses.

Utilizou-se um ensaio clínico "cross over" (duplo cego), e, como eixo de montagem, um estudo longitudinal em que o fator em estudo foi a infiltração do NOM com soro fisiológico 0,9\% (SF) e bupivacaína 0,5\% (BP). Conforme a droga utilizada, os pacientes foram divididos em dois grupos: 1) grupo SF-BP quando utilizaram o SF como primeiro bloqueio anestésico e BP durante o segundo bloqueio; 2) grupo BP-SF quando utilizaram BP no primeiro e SF no segundo bloqueio anestésico. O efeito clínico foi quantificado subjetivamente através de escala visual analógica da dor (VSA) onde se avaliou o número, duração e intensidade das crises. Objetivamente avaliou-se os limiares de percepção dolorosa, conforme técnicas de algometria ${ }^{4,5}$, estabelecendo-se um protocolo de pesquisa sobre o NOM, região temporal e sobre o primeiro, segundo e terceiro ramos do trigêmeo (nervos supra-orbital, infra-orbital e mental respectivamente) bilateralmente ${ }^{6}$.

$O$ estudo desenvolveu-se a partir de quatro consultas médicas que antecederam os períodos "run in", primeiro e segundo "washout" e análise final. Na primeira visita e em todas as outras três, os pacientes foram submetidos a anamnese, exame físico e neurológico. Após estes procedimentos todos os pacientes admitidos na primeira visita foram alocados para período "run in" de 30 dias, quando utilizaram medicamentos placebo, denominado de cidralina (200mg de amido) como proposta de tratamento profilático durante 30 dias. Este mesmo tratamento foi utilizado nos 30 dias do primeiro e do segundo período de "washout". Eventuais crises de migrânea, durante esta fase, no primeiro e no segundo período de "washout" foram tratadas com succinato de sumatriptano $50 \mathrm{mg}$ como dose inicial e podendo a critério do paciente chegar até uma dose máxima de $100 \mathrm{mg} /$ crise. Durante todas as fases do estudo, fichas contendo a VSA foram entregues aos pacientes para preenchimento em eventuais crises de migrânea, avaliando-se o padrão biológico das crises para cada paciente estudado. Na segunda visita, após exame clínico e neurológico, todos os pacientes que preencheram adequadamente a VSA foram submetidos a algometria. Após estes procedimentos realizou-se a infiltração do NOM bilateralmente (soro fisiológico ou bupivacaína, conforme sorteio). Na terceira visita médica, todos os pacientes que preencheram adequadamente a VSA foram submetidos a anamnese, exame físico, neurológico e algometria. Após estes procedimentos realizou-se a infiltração do NOM bilateralmente (soro fisiológico ou bupivacaína, ou seja o oposto do primeiro bloqueio anestésico). $\mathrm{Na}$ quarta consulta ou visita final, todos os pacientes que preencheram adequadamente a VSA foram submetidos a anamnese, exame físico, neurológico e algometria final, encerrando desta maneira a captação de dados. Foram selecionados voluntários não portadores de cefaléia nos últimos 12 meses, para compor o grupo controle algométrico.

Dos sessenta e três pacientes admitidos no estudo, 37 o completaram, sendo 8 do sexo masculino $(21,6 \%)$ e 29 do sexo feminino $(78,4 \%)$, com idade média de 37,3 (+ 8,2 anos. Iniciaram o estudo no grupo (BP-SF) 20 pacientes, três homens (15\%) e 17 mulheres (85\%), com idade média de 37,1 anos e 17 no grupo (SF-BP), quatro homens $(23,5 \%)$ e 13 mulheres $(76,5 \%)$, tendo idade média de 37,6 anos. Vinte e três pacientes foram excluídos por não apresentarem hipoestesia no território do NOM em pelo menos uma das duas infiltrações $(n=3)$, não comparecimento em uma das consultas $(n=16)$, síncope durante a infiltração $(n=1)$, utilização de outro medicamento durante o protocolo $(n=2)$, aparecimento de outra forma de cefaléia durante o estudo (cefaléia secundária a sinusiopatia) e número de crises inferior a uma durante o período "run in" $(n=3)$.

Para comparação dos dados do grupo controle (algometria) com os grupos estudo e entre eles, bem como para avaliação do número de crises, duração e intensidade entre os grupos estudo, utilizou-se os testes paramétrico de "t de Student" e o não paramétrico "Qui' Quadrado", e a Análise da Variância (ANOVA), para amostras independentes e relacionadas. O nível de significância (ou probabilidade de significância) mínima adotado foi de $5 \%(0,05)$. O protocolo de estudo foi submetido a avaliação e aprovado pelo Comitê de Ética Médica e Pesquisa em Seres Humanos da Universidade Federal do Paraná, Hospital de Clínicas.

\section{RESULTADOS}

Na comparação do grupo controle com o grupo estudo (fase run in), em relação à avaliação algométrica, foi constatado que os pacientes do grupo estudo apresentaram valores algométricos inferiores do lado direito para os nervos mental $(p=0,008)$ e supra-orbital $(p=0,017)$ e do lado esquerdo, para o nervo supra-orbital $(p=0,022)$. As demais comparações algométricas não apresentaram diferenças estatísticamente significativas (Tabela 1). Nos grupos e entre os grupos (BP-SF) e (SF-BF), a análise dos valores algométricos, nos diferentes pontos e em diferentes fases (antes da primeira, após a primeira e após a segunda infiltração), não mostraram diferenças estatisticamente significativas.

Os 20 pacientes do grupo (BP-SF), apresentaram em média no período "run in": 3,6 crises com duração de 1,8 dias e intensidade de $56 \mathrm{~mm}$ na VSA. Após a primeira infiltração apresentaram em média 3,2 crises com duração de 1,7 dias e intensidade de 
Tabela 1. Estatística descritiva da avaliação algométrica do grupo controle e do grupo estudo (Fase "run-in").

\begin{tabular}{|c|c|c|c|c|}
\hline DADOS - Grupo Controle & Média & Mínimo & Máximo & Mediana \\
\hline \multicolumn{5}{|l|}{ Lado Direito } \\
\hline Occipital Maior & 145,0 & 56,0 & 339,0 & 135,0 \\
\hline Mental & 46,2 & 17,0 & 83,0 & - \\
\hline Supra-orbital & 47,7 & 31,0 & 70,0 & - \\
\hline Infra-orbital & 53,9 & 28,0 & 98,0 & 48,0 \\
\hline Temporo-auricular & 123,6 & 54,0 & 288,0 & 113,0 \\
\hline \multicolumn{5}{|l|}{ Lado Esquerdo } \\
\hline Occipital Maior & 143,4 & 45,0 & 339,0 & 131,0 \\
\hline Mental & 48,6 & 28,0 & 85,0 & - \\
\hline Supra-orbital & 45,2 & 27,0 & 80,0 & - \\
\hline Infra-orbital & 55,4 & 29,0 & 98,0 & 52,0 \\
\hline Temporo-auricular & 119,6 & 49,0 & 250,0 & 103,0 \\
\hline DADOS - Grupo Estudo & Média & Mínimo & Máximo & Mediana \\
\hline \multicolumn{5}{|l|}{ Lado Direito } \\
\hline Occipital Maior & 138,1 & 56,0 & 256,0 & 124,0 \\
\hline Mental & 63,0 & 30,0 & 153,0 & 52,0 \\
\hline Supra-orbital & 65,4 & 27,0 & 231,0 & 49,0 \\
\hline Infra-orbital & 64,7 & 25,0 & 144,0 & 54,0 \\
\hline Temporo-auricular & 119,8 & 43,0 & 212,0 & 107,0 \\
\hline \multicolumn{5}{|l|}{ Lado Esquerdo } \\
\hline Occipital Maior & 133,7 & 51,0 & 226,0 & 126,0 \\
\hline Mental & 60,6 & 27,0 & 215,0 & 48,0 \\
\hline Supra-orbital & 56,8 & 25,0 & 134,0 & 50,0 \\
\hline Infra-orbital & 61,9 & 27,0 & 163,0 & 51,0 \\
\hline Temporo-auricular & 120,8 & 46,0 & 208,0 & 110,0 \\
\hline
\end{tabular}

$6,4 \mathrm{~mm}$ na VSA. Após a segunda infiltração apresentaram em média 2,4 crises com duração de 1,4 dias e intensidade de 5,2 mm na VSA (Tabela 2 e Fig 1 ). A análise estatística individual neste grupo e durante os três períodos mostrou que para o número de crises não houve diferenças estatisticamente significativas nos três períodos. A duração das crises referentes ao período antes e após a primeira infiltração não apresentaram diferenças estatisticamente significativas, porém no período após a primeira e após a segunda infiltração a duração média das crises foi menor $(p=0,029)$. Para a intensidade da dor não observamos diferenças estatisticamente significativas no período antes da primeira e após a primeira infiltração, porém no período após a primeira e após a segunda infiltração as crises apresentaram uma redução em sua intensidade $(p=0,035)$.

Os 17 pacientes do grupo (SF-BP) apresentaram em média no período "run in" 3,6 crises com duração de 1,6 dias e intensidade de 59mm na VSA. Após a primeira infiltração apresentaram em média 2,8 crises com duração de 1,9 dias e intensidade de $58 \mathrm{~mm}$ na VSA. Após a segunda infiltração apresentaram em média 2,5 crises com duração de 1,5 dias e intensidade de $67 \mathrm{~mm}$ na VSA (Tabela 3 e Fig 1). A análise estatística individual neste grupo e durante os três períodos mostrou que para o número e intensidade media das crises não houve diferenças estatisticamente significativas nos três períodos. A duração das crises referentes ao período antes e após a primeira infiltração não apresentaram diferenças estatisticamente significativas, porém no período após a primeira e após a segunda infiltração a duração média das crises foi menor $(p=0,015)$.

A comparação entre os dois grupos, (BP-SF) versus (SF-BP) evidenciou que o número e a duração média das crises não foram estatísticamente significativos entre si nas três fases do estudo. A intensidade das crises no período antes da primeira e após a primeira infiltração não foram estatisticamente significati- 
Tabela 2. Estatística descritiva da escala visual do grupo de estudo, Grupo (BP-SF).

\begin{tabular}{lcccc}
\hline ANTES DA PRIMEIRA INFILTRAÇÃO & & & & \\
DADOS & Média & Mínimo & Máximo & Mediana \\
\hline Número de Crises & 3,6 & 1,0 & 8,0 & 3,0 \\
Duração da Dor (dias) & 1,8 & 1,0 & 3,0 & 2,0 \\
Intensidade da Dor & 5,6 & 2,2 & 10,0 & 5,2 \\
\hline APÓs A PRIMEIRA INFILTRAÇÃO & & & & \\
DADOS & Média & Mínimo & Máximo & Mediana \\
\hline Número de Crises & 3,2 & 1,0 & 6,0 & 3,0 \\
Duração da Dor (dias) & 1,7 & 1,0 & 3,0 & 2,0 \\
Intensidade da Dor & 6,4 & 2,4 & 10,0 & 5,7 \\
\hline APÓs A SEGUNDA INFILTRAÇÃO & & & & \\
DADOS & & & & \\
\hline Número de Crises & Média & Mínimo & Máximo & Mediana \\
Duração da Dor (dias) & 2,4 & 1,0 & 6,0 & 2,5 \\
Intensidade da Dor & 1,4 & 1,0 & 3,0 & 1,0 \\
\hline & 5,2 & 2,8 & 10,0 & 3,9 \\
\hline
\end{tabular}

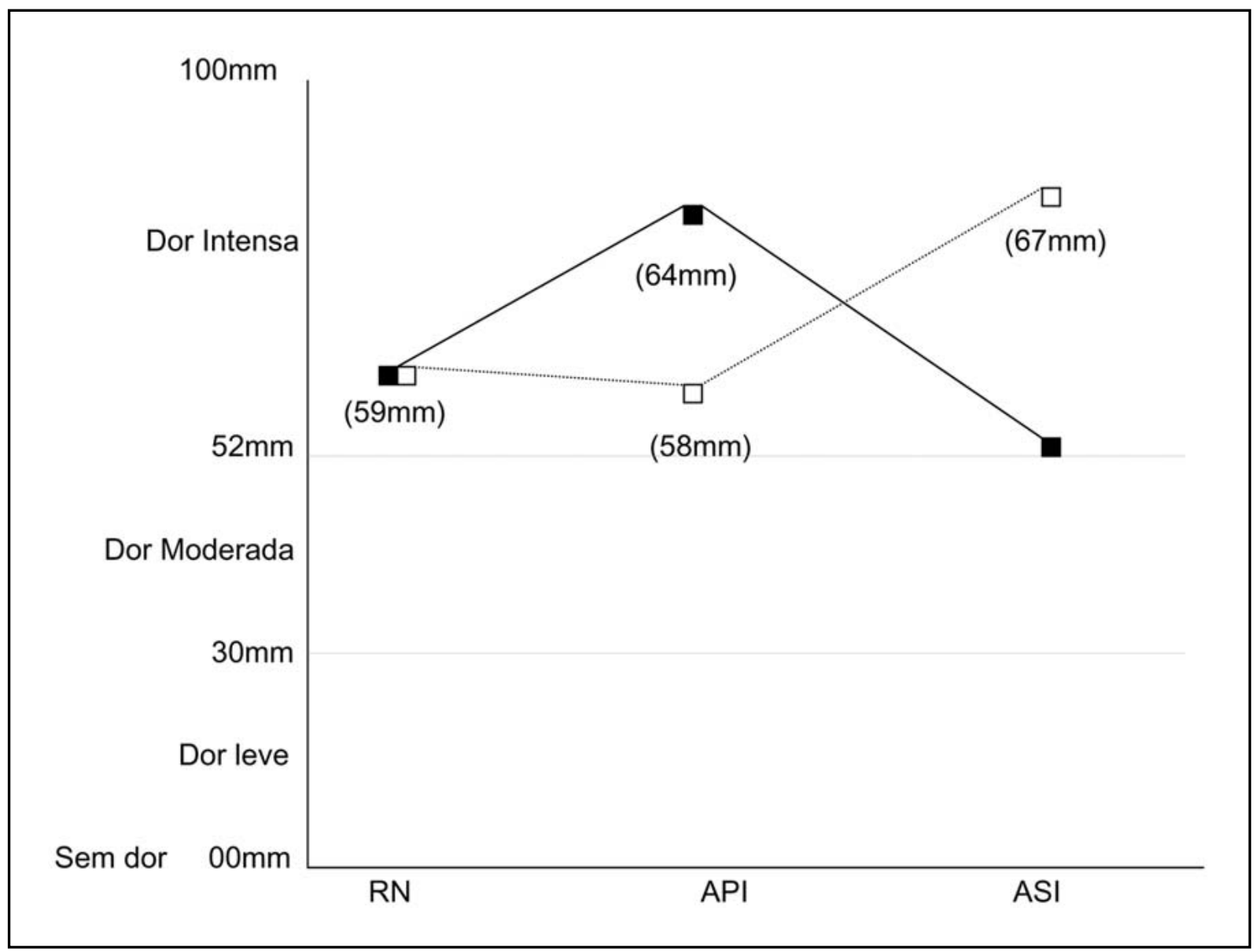

Fig 1. Intensidade das crises nas diferentes fases do estudo, período run in (RN), após a primeira infiltração (API) e após a segunda infiltração (ASI), para os grupos (BP-SF) n (SF-BP) $\square$. 
Tabela 3. Estatística descritiva da escala visual do grupo de estudo, Grupo (SF-BP).

\begin{tabular}{lcccc}
\hline ANTES DA PRIMEIRA INFILTRAÇÃO & & & & \\
DADOS & Média & Mínimo & Máximo & Mediana \\
\hline Número de Crises & 3,6 & 1,0 & 7,0 & 3,0 \\
Duração da Dor (dias) & 1,6 & 1,0 & 3,0 & 1,0 \\
Intensidade da Dor & 5,9 & 3,1 & 10,0 & 5,5 \\
\hline
\end{tabular}

\begin{tabular}{lcccc}
\hline APÓS A PRIMEIRA INFILTRAÇÃO & & & & \\
DADOS & Média & Mínimo & Máximo & Mediana \\
\hline Número de Crises & 2,8 & 1,0 & 6,0 & 2,0 \\
Duração da Dor (dias) & 1,9 & 1,0 & 3,0 & 2,0 \\
Intensidade da Dor & 5,8 & 2,7 & 10,0 & 5,3 \\
\hline
\end{tabular}

\begin{tabular}{lcccc}
\hline APÓS A SEGUNDA INFILTRAÇÃO & & & & \\
DADOS & Média & Mínimo & Máximo & Mediana \\
\hline Número de Crises & 2,5 & 1,0 & 6,0 & 2,0 \\
Duração da Dor (dias) & 1,5 & 1,0 & 3,0 & 1,0 \\
Intensidade da Dor & 6,7 & 3,0 & 10,0 & 7,4 \\
\hline
\end{tabular}

vos, porém no período após a primeira e após a segunda infiltração as crises apresentaram uma intensidade menor no grupo (BP-SF) $(p=0,020)$.

\section{DISCUSSÃO}

Poucos estudos mostraram, de maneira experimental, o efeito clínico obtido após a infiltração do NOM durante as crises de migrânea ${ }^{7-9}$, não sendo encontrado, até o presente momento, nenhum estudo utilizando esta técnica como estratégia profilática. Pacientes portadores de migrânea sem aura, após serem submetidos à infiltração do NOM e do nervo supra-orbital (NSO) com 0,5 a $1 \mathrm{ml}$ de bupivacaína $0,5 \%$ (BP) durante as crises, apresentaram uma redução no número de ataques, no consumo de analgésicos, na duração e na intensidade das crises. A redução da sensitividade e um decremento nas informações nociceptivas centrais associadas a sua ação sobre a atividade simpática periférica (perivascular) parecem justificar os resultados encontrados $^{9}$. No entanto, outro estudo, com metodologia semelhante, mostrou que o bloqueio do NOM e do NSO $(1,5 \mathrm{ml}$ de lidocaína), durante as crises de cefaléia cervicogênica, do tipo tensional e migrânea não promovia redução no número, na intensidade e na duração das crises de migrânea ${ }^{8}$.

O número de crises apresentada por um migranoso está diretamente relacionado com os mecanismos etiopatogênicos. A influência de fatores externos e internos desencadeando crises demonstra que mais de uma estrutura cerebral esta envolvida ${ }^{10-12}$. A influência positiva do NOM sobre esses mecanismos e sobre o número de crises foi comprovada nos estudos de Caputi ${ }^{9}$ e descartada nos estudos de Bovim ${ }^{7}$. No nosso estudo a comparação entre ambos os grupos, (BP-SF) versus (SF-BP), não mostrou redução no número de crises.

Após o início dos eventos geradores do desequilíbrio nociceptivo central e consequente surgimento da migrânea, uma verdadeira disputa entre forças nociceptivas irá surgir. Mecanismos de inibição central e periférica aparecerão em diferentes sítios cerebrais na tentativa de restabelecer este equilíbrio ${ }^{13-21}$. O tempo necessário para que isto ocorra, corresponde a duração média das crises de migrânea. Estudos anteriores mostraram que a duração das crises podem alterar, após a infiltração do NOM e do NSO', enquanto outros não mostraram diferenças ${ }^{7}$. Em nosso estudo não encontramos diferenças estatisticamente significativas que comprovem a influência do NOM sobre a duração média das crises.

Estímulos antidrômicos, supracorticais, podem chegar às terminações livres dos nociceptores promovendo a liberação de neurotransmissores (substância P, peptídeo relacionado ao gene da calcitonina e neurokinina A). Estes neurotransmissores, além de serem liberados na membrana sináptica, irão também produzir um fluxo retrógrado de informações nociceptivas para o córtex cerebral. O núcleo caudal do trigêmeo por ativação dos receptores do N-metil- 
D-aspartato potencializara estes estímulos através dos mecanismos de "wind up" ou hiperexcitabilidade neuronal22. O córtex posterior do cíngulo (CPC) em suas áreas 23 e 31 de Brodmann também poderá produzir amplificação nociceptiva ${ }^{23,24}$. Outras estruturas podem atuar indiretamente sobre o CPC, por meio de uma rede funcionante que monitoriza e integra os estímulos sensoriais, como a substância cinzenta periventricular do mesencéfalo e substância cinzenta periaquedutal ${ }^{25,26}$. Estudos anteriores mostram que a infiltração do NOM e do NSO, durante as crises de migrânea, podem ou não reduzir sua intensidade $7,8,9$.

Neste estudo observou-se que o comportamento álgico dos pacientes migranosos após a infiltração do NOM com BP e SF possuem características próprias (Fig 1). Os pacientes de ambos os grupos (BP-SF) e (SF-BP) apresentaram um aumento na intensidade de suas crises após a infiltração com BP e uma redução após a infiltração com SF. Estes dados demonstram que a BP produziu um aumento na intensidade média das crises em qualquer um dos grupos (BP-SF) e (SF-BP) durante os 30 dias após a sua administração, e uma redução na intensidade média das crises após 60 dias de sua administração (somente o grupo (BP-SF) foi acompanhado por um período de 60 dias após a infiltração com BP). $O$ desconhecimento do comportamento álgico induzido pela infiltração do NOM com BP proporcionou um "bias" no grupo (SF-BP). Este grupo foi acompanhado somente por um período de 30 dias após o uso da $\mathrm{BP}$, ao passo que o efeito deste fármaco sobre a nocicepção necessita, pelos nossos achados, de um período com 60 dias de acompanhamento. Observamos que a BP pode potencializar a intensidade das crises de migrânea nos 30 dias imediatos a sua administração. Os mecanismos responsáveis por este padrão são desconhecidos até o presente momento. Pacientes com cefaléia cervicogênica, submetidos ao bloqueio anestésico com BP, apresentaram padrão semelhante (aumento da intensidade da dor) até dois dias após a infiltração do NOM, padrão este denominado pelo autor como padrão "til"27.

Pós sinápticamente, os receptores NMDA medeiam a despolarização das fibras do tipo $C$, que produzem ativação do cordão espinhal em ratos, e controlam a liberação de substância P nas lâminas I e II do corno dorsal espinhal cervical ${ }^{28,29}$. A ativação dos receptores do NMDA produzem também estímulos sobre os neurônios excitatórios nociceptivos trigemino-talámicos que medeiam a nocicepção dentro do núcleo caudal do trigêmeo ${ }^{30}$. O bloqueio do NOM (fibras do tipo C) reduz a possibilidade da influência sensorial sobre os neurônios dinâmicos de amplo alcance $^{31}$ e, assim, sobre os receptores do NMDA. Desta maneira ocorrerá uma redução dos mecanismos de "wind up", diminuição da liberação de substância P nas lâminas I e II do corno dorsal espinhal cervical, redução dos estímulos excitatórios sobre os nociceptores trigemino-talámicos e conseqüente redução na intensidade da dor. Estudos experimentais, utilizando-se de técnicas de "c-fos", mostraram que sua atividade ao nível do núcleo caudal do trigêmeo e dos primeiros seguimentos cervicais $\left(C_{1}\right.$ e $C_{2}$ ), após estímulo elétrico sobre o seio sagital superior em gatos, diminuiu com a utilização do MK801 , um potente antagonista dos receptores do NMDA $^{32}$.

A ausência de mecanismos de convergência, não especificamente ao nível dos receptores do NMDA, foi descartada por estudos recentes ${ }^{2,33-35}$. Se os mecanismos de convergência existem, esta convergência poderia ser inibitória? Especulamos que as fibras do tipo $\mathrm{C}$, oriundas do NOM, após convergirem para o núcleo caudal do trigêmeo (receptores do NMDA), apresentarão efeito inibitório. Quando o NOM é bloqueado, os seus efeitos inibitórios sobre os receptores do NMDA ao nível do núcleo caudal do trigêmeo desaparecem, potencializando os mecanismos de "wind up" do trigêmeo. Isto permitiria que o sistema trigeminal ficasse livre de seu antagonista natural, promovendo um acréscimo na intensidade das crises de migrânea dentro de 30 dias após a infiltração. Recentemente sugeriu-se que o metabolismo encontrado no núcleo caudal do trigêmeo após estímulos do NOM poderia não estar relacionado com mecanismos de convergência excitatória, porém significaria um aumento da atividade metabólica de neurônios inibitórios. Estes achados e estas hipóteses, como o próprio autor sugere, ainda são especulativos, não havendo nenhum evidência satisfatória que as comprove experimentalmente ${ }^{2}$.

Uma vez que a ação direta da BP não pode estar relacionada com o córtex do cíngulo, o seu efeito poderá ser sobre o NMDA ou qualquer outra estrutura ao nível do tronco cerebral . Os verdadeiros mecanismos que levaram aos achados deste estudo não podem ser comprovados, porém evidências, aqui apresentadas, sugerem o envolvimento de mecanismos mais complexos dentro do núcleo caudal do trigêmeo dos que até aqui conhecidos.

$\mathrm{O}$ presente estudo permite concluir que o bloqueio anestésico com BP sobre o NOM não altera, de maneira profilática, o número e a duração das 
crises, porém altera a intensidade das mesmas após 60 dias do seu bloqueio. O NOM participa ativamente da modulação nociceptiva craniana, influenciando desta maneira a intensidade das crises de migrânea sem aura.

\section{REFERÊNCIAS}

1. Vital JM, Grenier F, Dautheribes M, Baspeyre H, Lavignolle B, Sénégas $\mathrm{J}$. An anatomic and dynamic study of the greater occipital nerve (n. of Arnold): applications to the treatment of Arnold's neuralgia. Surg Radiol Anat 1989;11:205-210.

2. Goadsby PJ, Knight YE, Hoskin KL. Stimulation of the greater occipital nerve increases metabolic activity in the trigeminal nucleus caudalis and cervical dorsal horn of the cat. Pain 1997;73:23-28.

3. Headache Classification Comittee of the International Headache Society. Classification and diagnostic criteria of headache disorders, cranial neuralgias and facial pain. Cephalalgia 1988;8(Suppl-7):17-27.

4. Bovim G. Cervicogenic headache, migraine, and tension-type headache: pressure-pain threshold measurements. Pain 1992;51:169-173.

5. Sand T, Zwart JA, Helde G, Bovim G. The reproducibility of cephalic pain pressure thresholds in control subjects and headache patients. Cephalalgia 1997; 17:748-755.

6. Piovesan EJ, Tatsui CE, Kowacs PA, Lange MC, Pacheco C, Werneck LC. Utilização da algometria de pressão na determinação dos limiares de percepção dolorosa trigeminal em voluntários sadios: um novo protocolo de estudos. Arq Neuropsiquiatr 2001;59:92-96.

7. Bovim G, Sand T. Cervicogenic headache, migraine without aura and tension-type headache: diagnostic blockade of greater occipital and supra-orbital nerves. Pain 1992;51:43-48.

8. Caputi CA, Firetto V, Luzi FM. Il blocco anestetico del nervo grande occipitale nelle cefalee primarie: considerazioni a proposito di quattro casi di coomplessa interpretazione. Confinia Cephalalgica 1994;1:27-33.

9. Caputi CA, Firetto V. Therapeutic blockade of greater occipital and supraorbital nerves in migraine patients. Headache 1997;37:174-179.

10. Gomersall JD, Stuart A. Variations in migraine attacks with changes in weather conditions. Int J Biometerol 1973;17:285-299.

11. Henryk-Gutt R, Rees WL. Psychological aspects of migraine. J Psychossom Res 1973;17:141-153.

12. Peatfield RC, Glover V, Littlewood JT, Sandler M, Clifford RF. The prevalence of diet-induced migraine. Cephalalgia 1984;4:179-183.

13. Sessle BJ. The neurobiology of facial and dental pain: present knowledge, future directions. J Dental Res 1987;66:962-981.

14. Atweh SF, Dajani BM, Saadé N, Jabbur SJ. Supraspinal inhibition of trigeminal input into subnucleus caudalis by dorsal column stimulation. Brain Res 1985;348:401-404

15. Chiang CY, Dostrovsky JO, Sessle BJ. Periaqueductal gray matter and nucleus raphe magnus involvement in anterior pretectal nucleus-induced inhibition of jaw-opening reflex in rats. Brain Res 1991;544:71-78.

16. Dostrovsky JO, Hu JW, Sessle BJ, Sumino R. Stimulation sites in periaqueductal gray, nucleus raphe magnus and adjacent regions effective in suppressing oral-facial reflexes. Brain Res 1982;252:287-297.

17. Dostrovsky JO, Shah Y, Gray BG. Descending inhibitory influences from periaqueductal gray, nucleus raphe magnus, and adjacent reticular formation. II. Effects on medullary dorsal horn nociceptive and nonnociceptive neurons. J Neurophysiol 1983;49:948-960.
18. Sessle BJ, Hu JW, Dubner R, Lucier GE. Functional propierties of neurons in cat trigeminal subnucleus caudalis (medullary dorsal horn). II. Modulation of responses to noxious and non-noxious stimuli by periaqueductal gray, nucleus raphe magnus, cerebral cortex and afferent influences, and effect of naloxone. J Neurophysiol 1981;45:193-207.

19. Sotgiu ML. Inhibiton of the nociceptive jaw opening reflex by the lateral reticular nucleus (LRN) in the rabbit. Neurosci Lett 1986;65:145148.

20. Strassman A, Mason P, Moskowitz MA, Maciewicz R. Response of brainstem trigeminal neurons to electrical stimulation of the dura. Brain Res 1986;379:242-250

21. Tsobokawa T, Katayama Y, Hirayama T, Yamamoto T, Nishimoto H. Effects of thalamic sensory relay nucleus stimulation on the jaw-opening reflex in response to tooth-pulp stimulation in the cat. Appl Neurophysiol 1986;49:229-236.

22. Davis SN, Lodge D. Evidence for involvement of N-methyl-D-aspartate receptors in "wind up" of class 2 neurones in the dorsal horn of the rat. Brain Res 1987;424:402-406

23. Hsieh JC, Belfrage M, Stone-Elander S, Hansson P, Inguar M. Central representation of chronic ongoing neuropathic pain studied by positron emission tomography. Pain 1995;63:225-236.

24. Vogt BA, Derbyshire S, Jones AKP. Pain processing in four regions of human cingulate cortex localized with co-registered PET and MR imaging. Eur J Neurosci 1996;8:1461-1473.

25. Vogt BA, Finch DM, Olson CR. Functional heterogeneity in cingulate cortex: the anterior executive and the posterior evalutive regions. Cereb Cortex 1992;435-443.

26. Devinsky O, Morrel MJ, Vogt BA. Contributions of anterior cingulate cortex to behavior. Brain 1995;118:279-306.

27. Vincent MB, Luna RA, Scandiuzzi D, Novis SA. Greater occipital nerve blockade in cervicogenic headache. Arq Neuropsiquiatr 1998;56:720725 .

28. Nagy I, Woolf CJ. Lignocaine selectively reduces $C$ fibre-evoked neuronal activity in rat spinal cord in vitro by decreasing N-methyl-Daspartate and neurokinin receptor-mediated post-synaptic depolarizations: implications for the development of novel centrally acting analgesics. Pain 1996;64:59-70.

29. Marvizòn JC, Martinez V, Grady EF, Bunnett NW, Mayer EA Neurokinin 1 receptor internalization in spinal cord slices induced by dorsal root stimulation is mediated by NMDA receptors. J Neurosci 1997;17:8129-8136.

30. Wang XM, Mokha SS. Opiods modulate N-methyl-D-aspartic acid (NMDA)-evoked responses of trigeminothalamic neurons. J Neurophysiol 1996;76:2093-2096.

31. Mendell LM, Wall PD. Response of single dorsal cordal cells to peripheral cutaneous unmyelinated fibres. Nature 1965;206:97-99.

32. Classey JD, Knight YE, Goadsby PJ. MK-801 reduces Fos-like immunoreactivity in trigeminocervical complex of cat. Cephalalgia 1999; 19:394.

33. Sjaastad O, Fridriksen TA, Stolt-Nielsen A. Cervicogenic headache, $\mathrm{C}_{2}$ rhizopathy, and occipital neuralgia: a connection? Cephalalgia 1986, 6:189-195.

34- Pfaffenrath V, Damdelar R, Pollman W. Cervicogenic headache. The clinical picture, radiological findings and hypotheses on its pathophysiology. Headache 1987;27:495-499.

35. Piovesan EJ, Werneck LC, Teive HG, Navarro F, Kowacs PA. Neurofisiologia álgica na irritação tentorial: descrição de um caso secundário a meduloblastoma. Arq Neuropsiquiatr 1998;56(3-B):677-682. 